\title{
La gestión pública de una obra hidráulica compleja, entre el marco internacional y los intereses regionales privados: el caso del trasvase Tajo-Segura (España)
}

\section{LuIs Alfonso Escudero Gómez, Alejandro Martín Trigo*}

\section{RESUMEN}

Los procesos económicos globales han agravado los problemas, conflictos y tensiones derivados del uso del agua dulce a escala global, estatal, regional y local. En la época contemporánea, la aceleración derivada de la industrialización y la urbanización ha aumentado el consumo del agua y las infraestructuras artificiales para su utilización. Hoy, el capitalismo de mercado global se ha ido apropiando de los recursos naturales y en el uso del agua predominan los intereses privados.

Al mismo tiempo, la degradación medioambiental obliga a intentar compatibilizar el consumo humano y económico con el respeto hacia la naturaleza. En la legislación vigente, en áreas como la Unión Europea, ya se da prioridad a la conservación del medio ambiente sobre la utilización incontrolada del agua como recurso productivo, sobre todo a partir de la aprobación de la Directiva Marco del Agua en 2000. Sin embargo, la política hidráulica española se ha centrado en el agua como bien económico. Con esta premisa se construyeron infraestructuras como el trasvase Tajo-Segura.

Actualmente, España, como miembro de la UE, debe adaptarse a la DMA y esto genera un tensa relación internacional-nacional-regional por la gestión pública del trasvase. El artículo se centra en esta cuestión. Se aplicará una metodología deductiva y basada en un análisis teórico crítico a partir del estudio bibliográfico y de la normativa vigente, del apoyo en datos cuantitativos y de las reflexiones de los autores.

Se concluye que el trasvase no se ajusta a la sostenibilidad ambiental y ecológica que proclama la DMA, sino que sigue respondiendo a la necesidad de satisfacer la demanda de la cuenca receptora. Además, en periodos de sequía se permite el mercado de cesión de agua de la cuenca cedente a la receptora entre particulares, ignorando el impacto ecológico derivado y superponiendo los intereses económicos. La prioridad debería ser satisfacer la demanda de agua dulce como necesidad de abastecimiento para toda la población y evitar los impactos al medio ambiente. Sin embargo, es evidente que las autoridades públicas están principalmente preocupadas por satisfacer las demandas de agua que tienen que ver con la producción económica aun a sabiendas de no cumplir plenamente con la legislación europea. La contribución resultará de interés a nivel científico y social y se une al debate actual en torno a las políticas de agua dulce en las dinámicas globales.

\section{Palabras clave}

Directiva Marco del Agua; trasvase Tajo-Segura; usos del agua; política hidráulica; acumulación capitalista por desposesión.

\section{Tithe}

Public management of a complex hydraulic work, between the international frame and the private interests:The case of the Tagus-Segura river transfer in Spain

\begin{abstract}
Some global economic processes have aggravated the problems, conflicts and tensions that are derived from the use of freshwater on a global, state, regional and local scale. In modern times, the acceleration caused by industrialisation and urbanisation has increased water consumption, as well as the artificial infrastructures for its use. Nowadays,
\end{abstract}

DOI:

https://doi.org/I0.15366/relacionesinternacionales2020.45.0I5

Formato de citación recomendado:

ESCUDERO GÓMEZ, Luis Alfonso y MARTÍN TRIGO,Alejandro (2020).“La gestión pública de una obra hidráulica compleja, entre el marco internacional y los intereses regionales privados: el caso del trasvase Tajo-Segura (España)", Relaciones Internacionales, $\mathrm{n}^{\circ} 45$, pp. 327-344.
* Luis Alfonso ESCUDERO GÓMEZ, Doctor en Geografía por la Universidad de Santiago de Compostela. Profesor Titular de Universidad de Departamento de Geografía y Ordenación del Territorio en el Grado de Humanidades y Patrimonio de la Facultad de Humanidades de Toledo de la Universidad de Castilla-La Mancha (España). Sus investigaciones se centran en estudios de geografía humana. Contacto: luisalfonso.escudero@ uclm.es

Alejandro MARTíN TRIGO, Estudiante de último curso en Humanidades y Patrimonio en la Facultad de Humanidades de Toledo de la Universidad de Castilla-La Mancha. Su línea de investigación se centra en el caso del trasvase Tajo-Segura en la realización de su Trabajo Fin de Grado. Contacto: alejandro.martin10@ alu.uclm.es

Recibido: 16/04/2020

Aceptado: 02/06/2020 
capitalism in the global market has taken ownership of natural resources and, in the use of water, private interests predominate. At the same time, environmental degradation urges human and economic consumption to be made compatible with the respect for nature. In current legislation, priority is given to environmental conservation regarding uncontrolled use of water as a productive resource, especially since the adoption of the Water Framework Directive in the European Union in 2000. However, the Spanish hydraulic policy has centred on water as an economic good. With this premise, certain infrastructures were built, such as the Tagus-Segura transfer. At present, Spain, as an EU member, must adapt to the WFD, so this generates a tense relationship regarding the public management of said transfer internationally, nationally and regionally. The matter of water distribution in Spain is a territorial issue between different autonomous communities where political strategies have a growing impact. National hydraulic policy has led to a politicisation which has confronted different regions, therefore resulting in water being at the moment a determinative factor of regionalist identity. Such political and territorial rivalries on a regional and national state have become even more complicated in the case of the Tagus-Segura transfer due to the obligation to apply the WFD from the EU legislation adopted in 2000. There is also the situation that the internal water management processes are part of a complex international scenario.

This article focuses on situations that intend to overrun the national and regional scale, towards an international and global one. In spite of the fact that the Tagus-Segura transfer is a Spanish hydraulic work, its use has been widely covered by the EU legislation, that is, the WFD, so there is a fraught relationship as a consequence of the public management of said transfer at the present time. For this reason, the first point that is addressed is whether the WFD is being implemented regarding the transfer or there is an infringement in its principles with a prevalence of economic interests in the catchment area. The second question which is discussed is the relation of asymmetric power between private and social interests in the transfer, and whether it responds to the global logic of a growing appropriation of natural resources with economic purposes.

The main objective of this article is to shed light on such questions. To do so, a deductive methodology is used, based on a theoretical and critical analysis of the existing literature and the current legislation, as well as quantitative data and the authors' considerations. The structure of the article is created according to this introduction, and it continues with a theoretical framework and the results, and a final conclusion.

The main findings of this study are: I) the Tagus-Segura transfer violates and does not respond to the basic principles of the Water Framework Directive of the EU, where the traditional objective of satisfying the demand of the catchment area and its economic interests take precedence over environmental sustainability and the ecological improvement of the transferring river basin, and 2) the water use in the transfer can be framed in the global neoliberal trend of appropriation of natural resources, where private interests dominate over the social interests in an asymmetrical relationship of power.

The transfer has become a controverted issue of great complexity, which has led to territorial tension provoked by political confrontation between regional governments due to a competitive dynamic within the autonomous state. At the same time, Spain has persistently made an infringement of the principles marked by the European WFD. It is true that the legislation context in which the transfer was made was very different to that of the present day. In fact, the Spanish legislation did not contemplate the evaluation of environmental impacts back then. The transfer was a tool of hydrological planification that considered water to be an economic resource meant to satisfy the demand as the main objective.As a consequence, this implied the degradation of water ecosystems and the quality of water itself, resulting in a reduction of the latter within the Tagus river basin.

The application of the WFD in Spain has contributed to coordinating the efforts in water management better, and it has bolstered research to determine the specific effects on water resources at a national and basin levels. Nevertheless, there has been a minimal incorporation of progress and new scientific knowledge regarding water planification and management strategies in the long term. At present, it is still necessary to progress in the Spanish water policy, as well as to improve its inadequate governance and diminish the pressure and impact on water bodies.

The insufficient compliance of the WFD by the Spanish legislation framework, which was initialised by its late transposition and continued with a lack of compromise with the environmental preference in water management that the directive imposes, influences directly the Tagus-Segura transfer. In reference to environmental sustainability, the economic demand has been privileged continually, and this has entailed an impediment when water extraction prevents the river Tagus from having a true ecological flow. The costs of the infrastructure have not recovered yet as the WFD dictates, as water rates are not included.Thus, private interests in the catchment area are favoured, as transferred water is easily accessible at a lower price, so it is indirectly subsidised. Finally, an unclear water market between particulars has been permitted, which lacks in transparency, allowing water to be shared during periods of drought between basins, when the normal transfer remains limited as dictated by the WFD.

The priority should be to satisfy the freshwater demand as a necessity to supply all the population and to avoid environmental impacts. However, it is evident that the authorities mainly care about satisfying the demands of water that are related to economic production, even though they do not knowingly abide by the European legislation. Imaginative solutions are needed in order to find alternatives so that the spirit of the WFD is respected and water protection prevails, and public interest is given priority over private interests, although this is not always at reach in the current political context. For this reason, future academic works must tackle this issue. The contribution can be of interest at a scientific and social level, and it links to the current debate regarding freshwater policies in global dynamics.

\section{KEYWORDS}

Water Framework Directive; Tagus-Segura transfer; water use; water policy; capitalist accumulation by dispossession. 


\section{$\mathrm{I}$}

\section{ntroducción}

Tradicionalmente, la sociedad ha demandado a sus gobernantes la disponibilidad del recurso del agua para satisfacer sus necesidades, ya sea para el abastecimiento humano o para la producción económica, y esto ha motivado la construcción de obras hidráulicas que lo hiciesen posible, como acueductos, canales, embalses, etc., casi siempre sin reparar en sus impactos sobre la naturaleza. En la época contemporánea, la aceleración derivada de la industrialización y la urbanización ha aumentado el consumo del agua y las infraestructuras artificiales para su utilización. Hoy, el capitalismo de mercado global ha dado lugar a conflictos territoriales entre estados por su uso, pero también dentro de ellos a escala regional y local, dado que los modelos productivos, ya sean agrícolas, industriales o turísticos, entre otros, exigen un elevado consumo de este apreciado recurso. Al mismo tiempo, la degradación medioambiental obliga a intentar compatibilizar el consumo humano y económico con el respeto hacia la naturaleza.

En la legislación vigente, en áreas como la Unión Europea (UE) y en múltiples estados del mundo, se antepone la conservación del medioambiente sobre la utilización incontrolada del agua como recurso productivo. No obstante, a pesar del avance que ha supuesto este cambio legislativo, el agua sigue considerándose principalmente como un factor de producción económica. El derecho del agua evoluciona bajo la influencia de rivalidades de poder político entre élites regionales, poderes autónomos y el estado, así como por las obligaciones internacionales (Salinas, 2013), que en el caso estudiado vienen marcadas por lo establecido por la UE.

La cuestión del reparto del agua en España es un problema territorial entre diferentes comunidades autónomas donde las estrategias políticas tienen un impacto cada vez mayor (Salinas y Loyer, 2013). La política hidráulica nacional ha conducido a una politización que ha enfrentado entre sía comunidades autónomas y que ha supuesto que el agua constituya un factor determinante de identidad regionalista (Salinas, 2013). De hecho, estos conflictos socioterritoriales por el agua son comunes entre las cuencas emisoras y receptoras de los trasvases del agua en múltiples partes del mundo (Zhuang, 2016). Sin embargo, estas rivalidades políticas y territoriales a escala nacional y regional (Saurí y del Moral, 200 I; Hernández-Mora et al. 2014) se han complicado en el caso trasvase Tajo-Segura, al igual que en otros casos europeos (Hill et al., 2002; Kallis et al., 2006), por la obligación de aplicar la Directiva Marco del Agua (DMA) de la UE aprobada en 2000 (del Moral et al., 20I4; Pita et al., 20I4). Se da la premisa señalada por Salinas (2013) donde los procesos internos de gestión del agua forman parte de un entorno internacional complejo.

El sureste de la península ibérica es una de las áreas más áridas del continente europeo por su clima mediterráneo subdesértico. La escasez de precipitaciones provoca una reducida disponibilidad de agua. Esta situación se agrava durante los veranos con el aumento de temperatura produciendo una evapotranspiración que supera en cantidad a las precipitaciones y esto se agrava con periodos cíclicos de sequía (Morales, Rico y Hernández, 2005). Sin embargo, esta zona tiene otras condiciones, tales como la insolación, la ausencia de heladas o la protección aerológica que pueden dar lugar a notables desarrollos agrícolas si se soluciona el déficit hídrico mediante el regadío. Hoy, esta zona cuenta con un sector agrícola estratégico, por sus niveles de producción y exportación, con importantes efectos en la economía regional y nacional (Martínez et al. 2016), pero con un desfase entre oferta y demanda del agua (Martínez y Esteve, 2000). El trasvase TajoSegura conecta la cuenca del río Tajo que desemboca en el océano Atlántico con la cuenca del 
Segura que vierte sus aguas al mar Mediterráneo, atravesando las cuencas de los ríos Guadiana y Júcar desde 1979.

El estudio académico del trasvase Tajo-Segura es ya amplio y la literatura académica resultante es muy abundante. Sin ser el objetivo una revisión bibliográfica completa y solo a través de una selección de obras bibliográficas, hay que señalar que el trasvase Tajo-Segura ha sido tratado ampliamente desde una perspectiva económica (Box, 1988; Gil, 1994; Melgarejo, 2000; San Martín, 20II) y medioambiental (Morales, Rico y Hernández, 2005; Bolinches, De Stefano y Paredes-Arquiola, 2020), o resaltando ambos aspectos (Box y Morales, 1994; Morote, Olcina y Rico, 2018; Hernández-Mora, 2019). Igualmente, a la literatura académica le ha preocupado la relación entre el trasvase y la política hidrológica estatal (Juárez, 199I; Calvo, 1999), así como dentro de los desequilibrios hidrográficos de España (Sandoval, 1989; Gil, 1992; Gil, Martínez y Gómez, 2018) o los debates políticos dentro del estado por el trasvase (Melgarejo, 1997). Otros autores se han centrado en cuestiones específicas del trasvase como el uso del agua y su valor (Gómez y Mata, 1999; Calatrava y Martínez-Granados, 2012), la sostenibilidad ecológica, social y económica de los regadíos del trasvase (Gómez, López y Montaner, 201 I; Morote, Olcina y Rico, 2017), sus orígenes y puesta en marcha (Pérez, 2009), su continuidad y futuro (Gómez, 2017; Morote y Rico, 20 I8), el abastecimiento del área urbana de Madrid (Gallego, 20I3a), etc.

En este contexto literario académico, el presente artículo se centra en cuestiones que pretenden rebasar la escala nacional y regional hacia una internacional y global.Aunque se trate de una obra hidráulica española, su uso aparece mediatizado por la legislación de la UE, es decir, por la DMA, y se genera una tensa relación por la gestión pública del trasvase en el presente. Por esta razón, la primera cuestión que se plantea es si se está aplicando la DMA en el trasvase o si hay un incumplimiento en sus principios rectores por una prevalencia de los intereses económicos de la cuenca receptora. La segunda cuestión que se aborda es la relación de poder asimétrico entre el interés privado y el interés social en el trasvase y si responde a la lógica global identificada por Harvey (2003) de una creciente apropiación de recursos naturales con fines económicos.

El responder a estas cuestiones es el objetivo principal del artículo. Para ello, se recurre a una metodología deductiva y basada en un análisis teórico crítico a partir del estudio bibliográfico y de la normativa vigente, del apoyo en datos cuantitativos y de las reflexiones de los autores.Así, al igual que Morote, Olcina y Rico (20l8), se han analizado los estudios previos y las normativas para realizar el estudio de caso.

La estructura del artículo parte de esta introducción, continúa con un marco teórico y los resultados y acaba con una conclusión. La contribución puede resultar de interés a nivel científico y social y se une al debate actual en torno a las políticas de agua dulce en las dinámicas globales. 


\section{Marco teórico}

\section{I.I. Del derecho internacional del agua a la Directiva Marco del Agua y su incumplimiento en España}

El derecho internacional general sobre la regulación del agua dulce presta una especial atención al principio de utilización equitativa y razonable (Espinosa, 2008). Según el Instituto de Recursos Mundiales, casi cuarenta países sufren ya un estrés hídrico severo $y$, además, está previsto que la demanda global de agua aumente un 50\% en 2050 (Kahna, 20 I7). A escala planetaria, la Asamblea General de las Naciones Unidas reconoció en julio de 2010 el derecho humano al agua y al saneamiento estableciendo que todos los seres humanos tengan acceso a una cantidad suficiente de agua para su uso y que sea segura, aceptable y asequible (ONU, 2020). Anteriormente, en 1997, la ONU había adoptado la Convención sobre el Derecho de los Usos de los Cursos de Agua Internacionales para Fines Distintos de la Navegación que, sin embargo, hasta 2014 no entró oficialmente en vigor (Movilla, 2014) y solo fue ratificada por treinta y seis estados. Según McCaffrey (2018) es de esperar que el número de estados crezca a lo largo del tiempo, de modo que se fortalezca un marco global que regule la asignación y la protección de los cursos de agua internacionales, aunque de momento sigue siendo una situación futura y no del presente.

La UE, tras un proceso político de 12 años iniciado en el Seminario Ministerial de Política del Agua en Frankfurt en 1988 (Kallis y Butler, 200I), llegó al acuerdo final sobre la propuesta de la Directiva Marco del Agua (Directiva 2000/60). Se tuvo que librar una batalla política para prácticamente todos los artículos de la propuesta. La DMA institucionaliza objetivos basados en ecosistemas y en procesos de planificación a nivel de la cuenca hidrográfica como base para la gestión de los recursos hídricos. Afecta a todos los países miembros de la UE y marca una importante tendencia hacia un enfoque basado en los ecosistemas para la política del agua y la gestión de los recursos hídricos (Kallis y Butler, 200I). El objetivo general es un buen estado y no deteriorado para todas las aguas (superficiales, subterráneas y costeras). El medio para lograrlo en las aguas de los ríos es la organización y planificación a un nivel hidrológico (la cuenca fluvial) y la implementación de una serie de medidas de control de la contaminación (Kallis y Butler, 200I). Además, la DMA comprende sistemas de monitorización, informes y evaluaciones con el fin de tener una visión más amplia e integral de los impactos del cambio climático en la UE y las acciones de adaptación en el sector del agua (Escribano et al., 2017).

La DMA cambió fundamentalmente la gestión del agua en todos los estados miembros de la UE, colocando la ecología acuática en la base de las decisiones de gestión. Su implantación ha tenido, sin embargo, éxitos y problemas (Hering et al., 2010). Así, la integración de la política del agua en otras políticas (por ejemplo, la agricultura) es un problema a gran escala en la UE (Escribano et al., 2017). En el lado positivo, hay que señalar que la DMA ha mejorado la gestión ambiental de los recursos acuáticos y ha desencadenado la reorganización de la gestión del agua por captaciones hidrológicas, en lugar de fronteras administrativas; ha sido un incentivo importante hacia la armonización de los métodos de clasificación y monitoreo y ha dado lugar a un cambio fundamental en los objetivos de gestión para garantizar la integridad de los ecosistemas. Sin embargo, los conceptos subyacentes de la DMA y, en particular, la forma en que se ha implementado en la práctica ha recibido grandes críticas de políticos, administradores del agua y científicos (Moss, 2009; Dufour y Piegay, 2009). Además, la implementación de la DMA ha 
sido, y sigue siendo, un importante desafío donde casi todos los estados miembros de la UE han dejado pasar un tiempo considerable para desarrollarla, obtener los datos necesarios y preparar planes de gestión de cuencas fluviales (Hering et al., 20l0). Recientemente la Comisión Europea estudió la modificación de la DMA, pero concluyó que era un pilar fundamental de la legislación medioambiental de la UE, que no debía modificarse y, por el contrario, se debía fortalecer su cumplimiento (WWF, 2019), un hecho que todavía está en proceso.

En el caso de España, la DMA no fue transpuesta al ordenamiento jurídico español hasta diciembre de 2003 a través de la Ley 26/2003 (MITECO, 2019). Esta falta de compromiso inicial con los objetivos y principios de la nueva política europea en materia de agua y una deficiente transposición posterior de la DMA han lastrado, desde el inicio, el proceso de implementación de esta en España (Hernández-Mora,20 I2).Así,la legislación española sigue manteniendo la satisfacción de las demandas como un fin primordial de la planificación frente a los objetivos de sostenibilidad ambiental prioritarios en la DMA. Las medidas de gestión del agua propuestas en España dentro de los planes de gestión de las cuencas fluviales todavía están muy orientadas al suministro, no tienen en cuenta completamente la reducción estimada de los aportes de agua en las diferentes cuencas fluviales y no se ha implementado la prueba climática de medidas, principalmente para grandes infraestructuras de agua planificadas (Escribano et al., 2017), como en el caso del trasvase Tajo-Segura. Además, los conflictos interterritoriales sobre la gestión de cuencas compartidas entre distintas comunidades autónomas, entre otros problemas, han dificultado enormemente el proceso (Hernández-Mora, 2012). De hecho, la UE sancionó a España por el incumplimiento de los plazos de la DMA (Oficina de Publicaciones de la UE, 20I2). Además, coincidiendo con la finalización del primer ciclo de planificación hidrológica 2009-20I5, la Comisión Europea (2019) emitió un informe con recomendaciones al estado español para su mejora indicando múltiples deficiencias y señalando mejoras para los siguientes planes hidrológicos.

La aplicación de la DMA en España ha contribuido a coordinar mejor los esfuerzos en la gestión del agua y ha impulsado la investigación para determinar los impactos específicos en los recursos hídricos a nivel nacional y de cuenca fluvial. Sin embargo, la incorporación de los avances de la investigación y el nuevo conocimiento científico sobre la planificación del agua y las estrategias de gestión a largo plazo ha sido, según Escribano et al. (2017), humilde. En el presente, sigue siendo necesario progresar en la política española de aguas, mejorar su inadecuada gobernanza y disminuir las presiones e impactos sobre las masas de agua (González, 2018).

\section{2. Espacios hídricos de poder asimétrico: interés privado/interés social}

El agua es un recurso esencial para todos los seres vivientes y no tanto un recurso productivo dedicado al desarrollo económico (La Calle, 20I5). Aun así, la propia DMA, aunque señale que el agua no es un bien comercial como los demás, sino un patrimonio que hay que proteger, destaca su valor económico (Kaika, 2003). Hay un choque entre los intereses públicos y privados en los espacios hídricos, con una relación muchas veces asimétrica que favorece a los segundos. A escala global, hay una tendencia a aumentar el papel del sector privado en la gestión de los recursos naturales (Castree, 2008) y en especial en la del agua. Se trata de un proceso de desposesión mediante las recientes olas de privatización y descolectivización de los recursos hídricos que han tenido lugar por todo el mundo (Swyngedouw, 2007). El discurso neoliberal enfrenta lo 
público a lo privado y la administración pública del agua a la gestión privada. Tras identificarla con el mercado libre, competitivo, transparente y con información perfecta y atribuirle cualidades beneficiosas, se concluye que la gestión privada es mejor o más eficiente que la pública y se postula la conveniencia de privatizar dicha gestión (Naredo, 2007).

A escala global, el desarrollo de las actividades económicas ha dado lugar a actuaciones hidráulicas para aumentar la oferta de agua que garanticen el incremento de los usos agrícolas, hidroeléctricos y urbanos (Tortajada, 2016). En España, el paradigma que ha dominado la política de aguas se ha caracterizado por la preponderancia del sector público en el desarrollo de infraestructuras hidráulicas y en la asignación de recursos hídricos (regadío y energía, entre otros) a precios subvencionados (Hernández-Mora y del Moral, 20l6). Por otro lado, en los últimos años se ha establecido un mercado de agua entre particulares que fortalecen todavía más la posición de poder de los intereses privados sobre el interés social. Naredo (2007) sintetiza perfectamente esta cuestión para el caso español:

"La realidad del agua en nuestro país plantea tres paradojas (...). Estas paradojas son: $I^{a}$ los mercados de agua no acostumbran a ser libres, competitivos, transparentes, ni perfectos..., ni sus resultados tienen por qué ser, por principio, recomendables; $2^{a}$ lo supuestamente público se encuentra parasitado por lo privado; $3^{\mathrm{a}}$ los intereses privados dominantes en el sector no parecen querer esos mercados de agua libres, competitivos, transparentes y perfectos" (Naredo, 2007, p. I).

Bakker (2002) señala que España ha realizado un proceso neoliberalizador incompleto en la gestión del agua ya que el estado sigue desempeñando un papel preponderante. Sin embargo, Hernández-Mora y del Moral (2016) demuestran que a través de una serie de reformas legales se ha incrementado el rol de los instrumentos de mercado - al igual que en otros lugares del mundo (Bauer, 2010) - y el papel del sector privado en la gestión y asignación de los recursos hídricos por su teórica mayor eficiencia y eficacia. La prevalencia del discurso de la escasez de agua ha servido para justificar la expansión de los mercados para la reasignación de recursos hídricos escasos (Hernández-Mora y del Moral, 2016), donde la compra y venta de agua responden a provechos privados por encima de un interés social público. Estos intereses privados empresariales buscan más la concesión y el monopolio que la libre competencia y sus negocios no aseguran la buena gestión integrada del agua y de los ecosistemas vinculados a ella (Naredo, 2007), sino que buscan una rentabilidad económica capitalista, es decir, obtener beneficios por encima de otros cualesquiera fines.

En este contexto, Naredo (2007) afirma que lo público se encuentra parasitado por lo privado. De este modo, se hace difícil la búsqueda del interés general (Garrido, 1999).Aquello que resulta ser eficiente desde el punto de vista económico no necesariamente supone un bienestar colectivo (Bromley, 1992). Según Oñate (2016), la situación actual de la gobernanza en los temas del agua en España no es adecuada para lograr una sostenibilidad ambiental en su gestión. Si, a cambio, hubiese un contexto institucional avanzado (Berbel, Gutiérrez y Giannoccaro, 20l6), habría mecanismos de control que impedirían los errores y abusos de los agentes privados. 


\section{Resultados}

\section{I. El trasvase Tajo-Segura en un encuadre internacional: el incumplimiento de la DMA}

La cuenca del Tajo - la cedente del trasvase - es obviamente internacional, pues su curso bajo y desembocadura están en Portugal. Delgado (2002) califica el caso de las cuencas hidrográficas hispano-lusas como "un buen ejemplo de gestión de recursos hídricos compartidos" (p. 30l). Las aguas ibéricas han sido objeto de tratados bilaterales desde mediados del siglo XIX (Barreira, 2008). Actualmente estas cuencas compartidas (Miño, Limia, Duero, Tajo y Guadiana) se rigen por el Convenio sobre Cooperación para la Protección y el Aprovechamiento Sostenible de las Aguas de las Cuencas Hidrográficas Hispano-Portuguesas que se firmó en la ciudad portuguesa de Albufeira en 1998 y que entró en vigor en 2000 (Barreira, 2008). El Convenio de Albufeira se elaboró en el momento en que se negociaba la Directiva Marco del Agua (DMA) de la UE y, aunque España y Portugal son miembros de la UE, donde rige el principio de primacía del derecho comunitario, el Convenio de Albufeira sigue siendo el principal instrumento para la cooperación en las cuencas hispanoportuguesas (Barreira, 2008). En el caso concreto del Tajo, España y Portugal deben realizar una gestión que satisfaga el siguiente volumen de caudales mínimos $2.700 \mathrm{hm}^{3} / a n ̃ o$ en la estación de control de aforo de Cedillo (Cáceres) en España y próximo a la frontera y de 4.000 hmªño en Ponte Muge, ya cerca de la desembocadura en Portugal (Barreira, 2008).

En España, las aguas del Tajo discurren por varias comunidades autónomas y por este motivo la gestión de sus aguas corresponde al estado según recoge el artículo 149.1.22 de la Constitución de 1978. Esta gestión se canaliza a través del Plan Hidrológico Nacional al que según la Ley 10/200 I corresponde resolver los desequilibrios hídricos existentes en el territorio nacional. Sin embargo, esta gestión ha estado principalmente encaminada a satisfacer la demanda del agua como recurso productivo (Morote y Rico, 2018). Por el contrario, la legislación europea, con la entrada en vigor de la DMA, ha venido a establecer la prioridad medioambiental. Esto supone cambiar el paradigma tradicional aplicado en la política y gestión del agua en España, aquel que dio lugar a la construcción de grandes obras hidráulicas como el trasvase Tajo-Segura.

El trasvase se ha convertido en una cuestión de gran complejidad y muy controvertida, llevando a tensiones territoriales que provocan conflictos políticos entre gobiernos regionales por una dinámica de competición dentro del estado autonómico (Hernández-Mora, 20I3). Al mismo tiempo, tiene lugar un incumplimiento sostenido por parte de España de los principios internacionales marcados por la DMA europea. Es cierto que el contexto legislativo en que fue realizado el trasvase fue muy diferente al actual. De hecho, la legislación española de la época no contemplaba la evaluación de impacto medioambiental. El trasvase fue una herramienta de la planificación hidrológica que consideraba el agua como un recurso económico que debía satisfacer la demanda como objetivo principal. Esto tuvo como consecuencia la degradación de los ecosistemas acuáticos y la calidad de las aguas (Hernández-Mora, 20I3), dando lugar a que esta sea baja en la demarcación hidrográfica del Tajo (Ferreira, 2013).

Ha sido la DMA la que ha obligado a un cambio en la política de gestión del agua en toda la UE y, por tanto, en España. Ha introducido como principal objetivo proteger y recuperar los ecosistemas acuáticos que son imprescindibles para garantizar la disponibilidad de agua para los 
seres humanos, y esto contrasta totalmente con el objetivo desarrollista con el que fue concebido, construido y abierto el trasvase. Es decir, la DMA supone un giro en la política de gestión del agua frente a la idea principal que primaba en España, que era satisfacer las demandas del agua (La Calle, 20I5). No obstante, a pesar de la transposición en la legislación española, Hernández-Mora (2013) considera que solo se han solapado las nuevas prioridades con los objetivos anteriores y esto impide que la nueva prioridad marcada por la DMA se cumpla de forma efectiva.

El Plan Hidrológico Nacional (PHN) se refiere al trasvase de esta forma:

"El trasvase se configura, en el marco de la presente Ley, como un importante instrumento vertebrador del territorio,evitando que zonas con déficits estructurales de recursos hídricos vean estrangulado y amenazado su desarrollo económico y social por la incertidumbre del suministro de agua, y garantizando que las cuencas cedentes no vean hipotecado el suyo como consecuencia del mismo, recibiendo adicionalmente una compensación destinada a actuaciones medioambientales vinculadas a los usos del agua" (Ley 10/200I, de 5 de julio, del $\mathrm{PHN})$.

Esta cita no está en sintonía con la DMA. No toma en consideración como objetivo prioritario la protección y restauración de los ecosistemas fluviales. Hernández-Mora (20l3) señala que la DMA prioriza el buen estado ecológico de las aguas por delante de la satisfacción de la demanda de agua, mientras que la idea del trasvase en el PHN es la contraria y se centra en la demanda y la búsqueda del desarrollo económico.

A raíz de las modificaciones necesarias para adaptarse a la DMA,las normas y procedimientos de explotación del trasvase Tajo-Segura han sido modificados y establecidos primero por la Ley 21/20I3 de Evaluación Ambiental y, más tarde, complementados por el Real Decreto 773/20I4 por el que se aprueban diversas normas reguladoras del trasvase por el acueducto Tajo-Segura y la Ley 2 I/20I 5 por la que se modifica la Ley 43/2003, de 2 I de noviembre, de Montes (Morote y Rico, 2018). Para la elaboración de estas nuevas leyes y normas de explotación ha sido fundamental el llamado pacto del Memorándum, un acuerdo entre el gobierno central (Ministerio de Agricultura, Alimentación y Medio Ambiente) y los autonómicos de la Comunidad Valenciana y la Región de Murcia, a los que posteriormente se adhirieron los de Castilla-La Mancha, Madrid y Extremadura. Es una evidencia de la difícil relación multiescalar de la gestión del agua en España, que debe cumplir las normas internacionales de la DMA al tiempo que se llegan a acuerdos políticos entre las comunidades autónomas y el gobierno central. El pacto fue firmado en marzo de 20I3, pero con la significativa ausencia de participación pública (Hernández-Mora, 2019), requisito indicado por la DMA que fue ignorado.

Como elementos más importantes que introduce la nueva legislación y normativa se pueden señalar que se eleva el umbral mínimo de trasvase, anteriormente fijado en $240 \mathrm{hm}^{3}$ por el Plan Hidrológico Nacional (Ley 10/200 I), a 400 hm $^{3}$ (Ley 2I/20I3) y, fundamentalmente, se fija una nueva regla de explotación a partir del año hidrológico 2014/I 5 en función de las existencias 
conjuntas en los embalses Entrepeñas y Buendía en la cabecera del Tajo a comienzos de cada mes. Se establecen tres tipos de niveles mensuales con los que se acordará la realización de los trasvases y un cuarto donde no es posible la transferencia de agua (ver figura I).

\section{Condiciones de explotación trasvase Tajo-Segura}

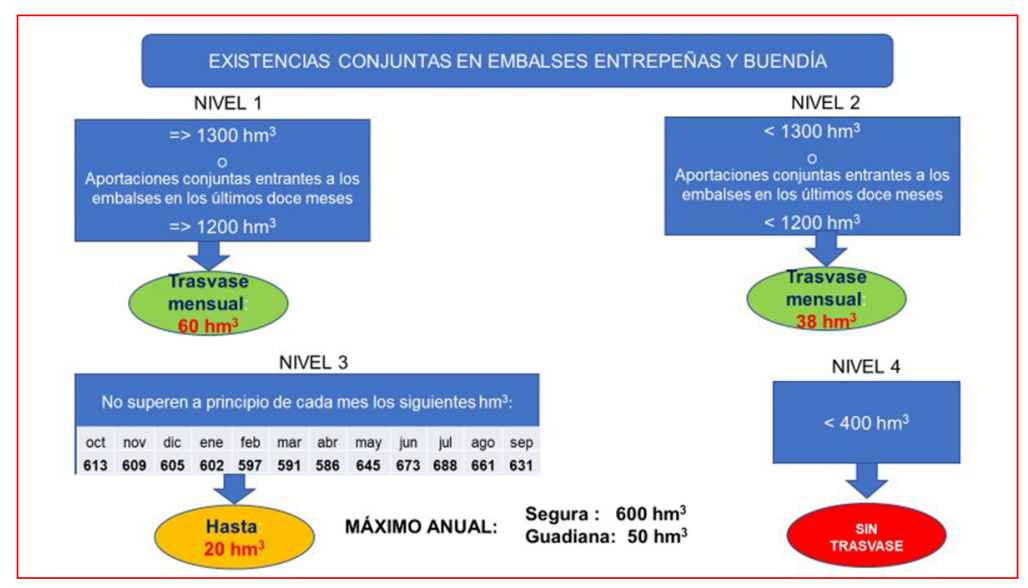

Fuente: Real Decreto 773/20I4, Ley 2//2015 y elaboración propia

En la tabla I se han trasladado las nuevas condiciones de explotación del trasvase a partir del agua embalsada existente Entrepeñas y Buendía desde el año hidrológico 2004/05 hasta el de 2007/I8. La razón es que el ciclo reciente entre 2004/05 y 2008/09 fue uno de los periodos de sequía más importantes de los últimos años. En él, según la normativa actual de explotación basada en una DMA que ya estaba aprobada desde el 2000, no se hubiera podido trasvasar agua en la mayoría de los meses. Sin embargo, se hicieron trasvases porque entonces el umbral mínimo establecido era de $240 \mathrm{hm}^{3}$ y, por tanto, se siguió suministrando agua a la cuenca beneficiaria. Es un largo periodo de tiempo donde, a pesar de existir ya la DMA, los criterios de sostenibilidad medioambiental fueron ignorados y prevalecieron los intereses económicos de los grupos de presión del sureste español. 
Tabla I.Agua embalsada en Entrepeñas y Buendía en los años hidrológicos del 2004/05 al 2017//8.

\begin{tabular}{|c|c|c|c|c|c|c|c|c|c|c|c|c|}
\hline $\begin{array}{l}\text { Año } \\
\text { hidrológico }\end{array}$ & oct. & nov. & dic. & ene. & feb. & mar. & abr. & may. & jun. & jul. & ago. & sep. \\
\hline $2004 / 05$ & 779 & 747 & 766 & 783 & 752 & 710 & 676 & 635 & 580 & 521 & 426 & 360 \\
\hline $2005 / 06$ & 332 & 298 & 286 & 301 & 305 & 298 & 328 & 348 & 340 & 309 & 265 & 244 \\
\hline $2006 / 07$ & 244 & 259 & 268 & 302 & 304 & 336 & 337 & 403 & 456 & $46 I$ & 408 & 361 \\
\hline $2007 / 08$ & 241 & 359 & 337 & 322 & 297 & 279 & 279 & 274 & 353 & 391 & 367 & 320 \\
\hline $2008 / 09$ & 310 & 315 & 315 & 349 & 395 & 487 & $55 I$ & 596 & 569 & 524 & 476 & 409 \\
\hline $2009 / 10$ & 374 & 375 & 360 & 380 & 648 & 813 & 994 & 1095 & 1166 & 1191 & 1195 & 1136 \\
\hline $2010 / 11$ & IIII & IIOI & 1096 & 1105 & II 57 & 1207 & 1274 & 1305 & 1301 & $|28|$ & 1184 & 1110 \\
\hline $2011 / 12$ & 1078 & 1073 & 1058 & 1040 & 1033 & 999 & 962 & 928 & 901 & 838 & 723 & 629 \\
\hline $2012 / 13$ & 590 & 590 & 596 & 566 & 579 & 629 & 712 & 940 & 991 & 978 & 882 & 797 \\
\hline $2013 / 14$ & 719 & 727 & 723 & 697 & 715 & 880 & 894 & 856 & 798 & 724 & 662 & 604 \\
\hline $2014 / 15$ & 536 & 543 & 552 & 532 & 535 & 569 & 581 & 586 & 527 & 468 & $42 I$ & 360 \\
\hline $2015 / 16$ & 331 & 332 & 330 & 315 & 346 & 434 & 504 & 575 & 627 & 586 & 544 & 474 \\
\hline $2016 / 17$ & 438 & 437 & 417 & 429 & 422 & 443 & 442 & 393 & 354 & 329 & 293 & 256 \\
\hline $2017 / 18$ & 236 & 238 & 233 & 235 & 238 & 266 & 467 & 668 & 691 & 702 & 656 & $6 / 4$ \\
\hline
\end{tabular}

* En negrita los meses con nivel 4 (periodo de sequía) y en cursiva los meses con nivel 3 (situación de excepción) establecidos desde el año hidrológico 2014/I5.

En realidad, solo cuando se alcanza el umbral de sequía se detienen los trasvases y este límite ampliado por criterios medioambientales, $400 \mathrm{hm}^{3}$, solo se ha considerado a partir de 20l4, pues anteriormente era menor. En el resto de las situaciones siempre ha prevalecido el criterio de necesidades económicas de la cuenca demandante sobre cualquier otro aspecto. Esto ha provocado que, con las oscilaciones derivadas del caudal embalsado, se haya mantenido un flujo persistente de agua del Tajo al Segura (ver figura 2).

\section{Evolución de los trasvases entre el Tajo y el Segura}

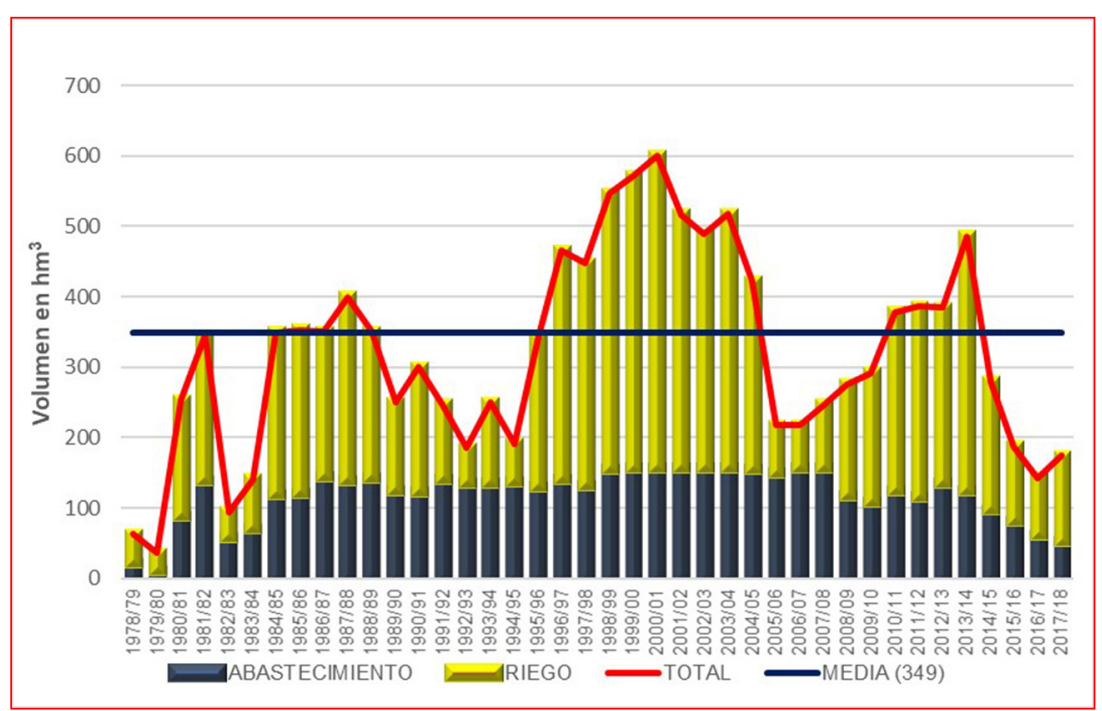

Fuente: CHS (2020) y elaboración propia. 
Por otro lado, la DMA introduce el concepto del buen estado ecológico para la evaluación de la estructura y funcionamiento de los ecosistemas acuáticos asociados a las masas de aguas superficiales (en las que se engloban los sistemas fluviales). Es un elemento que Baeza (20l3) considera como clave para analizar el estado de salud de los sistemas acuáticos. Una evidencia de la deficiente aplicación de la normativa europea es cómo se interpretan los indicadores establecidos por la DMA para determinar el estado ecológico de la cuenca del Tajo. Empleando una serie de medidores fisicoquímicos, hidromorfológicos y biológicos se determina si los tramos de una cuenca tienen un estado ecológico valorado con cinco referencias que van desde "muy bueno" a "malo" (Baeza, 20l3). Esta valoración es un instrumento para considerar si es necesario tomar medidas tanto preventivas como correctoras con el fin de alcanzar el buen estado de los ecosistemas. Sin embargo, Baeza (20l3) señala que se han detectado deficiencias porque los informes oficiales no tienen suficiente grado de detalle. Por esta razón, tramos que son calificados como "buenos" en realidad no lo son. La propia Comisión Europea, en referencia al estado ecológico y los métodos de evaluación empleados, realiza observaciones como la siguiente que demuestran la falta de rigor en las valoraciones:
"Resulta especialmente preocupante que el QEI-4 (fauna ictiológica) no haya sido desarrollado, ya que este $B Q E$ [elemento de calidad biológica] resulta especialmente pertinente para evaluar muchas de las presiones, en particular la captación de agua, la alteración hidrológica, los cambios morfológicos y la contaminación” (CE, 2019, p. 55).

El hecho es que el eje central del río Tajo presenta un grave empobrecimiento de especies y organismos, así como la sustitución de fauna autóctona por otra exótica, afectando tanto a los vertebrados como a los invertebrados (Yela, 20I3). Los peces autóctonos que tiene el Tajo hoy se encuentran amenazados por la contaminación, la ausencia de caudales, la destrucción de las riberas fluviales, el cambio climático y la introducción de especies exóticas (Gómez, 20l3). Igualmente, en la cuenca del Tajo existen Zonas de Especial Protección para las Aves (ZEPA) que se ven afectadas por el impacto medioambiental que sufre el río, como denunció el gobierno autonómico de Castilla-La Mancha (Diario ABC, 2017). Son muestras de una conclusión que es la gestión insostenible del Tajo derivada del incumplimiento sistemático de la DMA en su cuenca. En este sentido, se coincide con la afirmación de San Martín et al. (20I5, p. I):

"La planificación y gestión del agua en dicha demarcación no es sostenible en ninguna de las tres principales dimensiones del desarrollo sostenible (económica, social y ambiental) y, lo que es más grave, las instituciones que la gestionan (dimensión política-institucional o de gobernanza) no han asumido en sus políticas que la sostenibilidad es un objetivo obligatorio de primera magnitud. La clara consecuencia de este hecho es el incumplimiento sistemático de la Directiva Marco de Agua europea en la cuenca del Tajo". 


\section{2. El trasvase Tajo-Segura en un encuadre global: la prevalencia del interés privado capitalista}

Frente a las directrices internacionales, la sostenibilidad no es la matriz rectora del trasvase TajoSegura, sino los intereses económicos privados que se benefician de esta gran infraestructura hidráulica. Las políticas que han surgido en los últimos años sobre la preocupación del estado del agua, como señalan Hering et al. (2010) para la UE, a menudo se enfrentan con fuertes afanes económicos que se oponen a una difusa idea del interés público en general. En el caso del trasvase, esos intereses son los beneficiarios del agua del trasvase, la demanda de la cuenca receptora.

Morote, Olcina y Rico (2018) han calculado que el $86 \%$ de la demanda del agua de la cuenca receptora, la del Segura, es para riego. Su gestión se canaliza a través del Sindicato Central de Regantes del Acueducto Tajo-Segura (SCRATS), que es el único representante del colectivo de regantes y que tiene como objetivo principal defender los intereses de todos sus miembros: las comunidades de regantes y usuarios titulares de los derechos al riego con agua del trasvase (Pérez, 2009). Forman un lobby activo en el empleo del trasvase que presiona constantemente para su utilización en su beneficio. Su argumento más poderoso es el valor del uso del agua en la cuenca receptora con respecto a la cedente. Calatrava y Martínez-Granados (2012) estimaron que el regadío de la cuenca del Segura genera una rentabilidad media privada, medida en términos

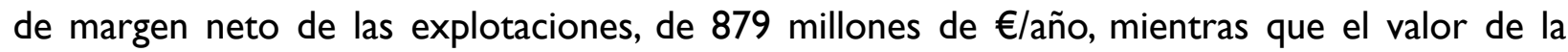
aportación media anual de los recursos trasvasados desde la cabecera del Tajo sería de 169 millones de euros. En estos cálculos aparecen las dos realidades que motorizan el trasvase en el presente y desde su origen: los beneficios productivos económicos que se generan y los intereses privados de los beneficiarios de este recurso.

Además del agua demandada para regadío, siguiendo de nuevo a Morote, Olcina y Rico (2018), el II\% de la restante se correspondería a los usos urbanos, siendo este su segundo gran uso. Una demanda de agua muy concreta, relacionada con el desarrollo turístico e inmobiliario del sureste español, es la de los campos de golf que los autores calculan en un 0,65\%. En definitiva, con el trasvase se atiende el interés de las demandas agrícolas, principalmente, así como urbanas y turísticas en un área con escasez natural de recursos hídricos (Rico et al., 20l3). Estamos ante una apropiación de recursos naturales con fines económicos, lo cual es según Harvey (2003) una evidencia más de la acumulación capitalista privada por desposesión de lo común.

Los usuarios del agua trasvasada han de pagarla mediante las tarifas establecidas. Según la Ley 52/1980 de Regulación del Régimen Económico de la Explotación del Acueducto TajoSegura, los conceptos que componen la tarifa del agua trasvasada son la amortización del coste de las obras, los gastos fijos de funcionamiento y los variables de funcionamiento. En realidad, sin embargo, las tarifas en la práctica se han calculado simplemente según el volumen real de agua trasvasada (Gallego, 2013b). En ellas no se ha considerado realmente la recuperación de costes medioambientales y relacionados con los servicios del agua, como estipula la DMA (HernándezMora, 20I3).Así, en las tarifas no se tienen en cuenta conceptos como el valor real de las obras, los costes ambientales o aquellos relacionados con la contaminación. Los precios del agua trasvasada han favorecido así a los compradores privados de la cuenca receptora hasta el punto de que Hernández-Mora y del Moral (2016) hablan de aguas subvencionadas. 
Por otro lado, existe un mercado del agua entre comunidades regantes de la cuenca cedente y de la receptora que la legislación española permite o, al menos, no prohíbe directamente. Son contratos formales de cesión de agua entre particulares realizados en el trasvase Tajo-Segura y representan el mayor volumen de cesiones de derecho de agua en España hasta la fecha (Palomo y Gómez, 2016). En ellos, comunidades regantes del Alto Tajo actúan como vendedoras y, principalmente el SCRATS, como comprador mediante una contraprestación económica. Este tipo de intercambios son habituales en los periodos de sequía. Existen, igualmente, contratos de opción, de mayor flexibilidad, donde las partes contratantes adquieren el derecho (opción), pero no la obligación, de venta o adquisición del agua a un precio prefijado para una fecha determinada (Palomo y Gómez, 2016).

Mediante estos contratos, el mercado del agua actúa como una institución reasignadora de recursos en España (Palomo y Gómez, 20l4). Es una satisfacción para la demanda de los usuarios agrarios y urbanos de la cuenca del Segura durante épocas de sequía, pues en periodos donde el trasvase funciona normalmente, los intercambios se han reducido al mínimo (Palomo y Gómez, 2016). Suponen una elevada cantidad de recursos en el Segura resuelta mediante acuerdos entre particulares, con cierta opacidad (Palomo y Gómez, 2016), que escapan a la gestión pública y cuya raíz son los intereses económicos de compradores y vendedores. Benefician a las partes contratantes por encima del interés general. Las implicaciones que estos contratos suponen sobre la sostenibilidad y los valores ecológicos de la cuenca del Tajo son evidentes, pues las cesiones se realizan en los periodos de sequía. Los mercados son un alivio permitido para los intereses económicos privados por encima de un interés social general y de los principios de la DMA. Han servido para intensificar la presión sobre los ecosistemas del Tajo (Hernández-Mora y del Moral, 2016). Una lógica neoliberalizadora de carácter global se impone a la medioambiental.

\section{Conclusión}

Como conclusión principal del artículo se apuntan las respuestas a las dos cuestiones planteadas en sus objetivos: I) el trasvase Tajo-Segura incumple y no responde a los principios básicos de la Directiva Marco del Agua de la UE, prevaleciendo el objetivo tradicional de satisfacer la demanda de la cuenca receptora y sus intereses económicos sobre la sostenibilidad ambiental y la mejora ecológica de la cuenca hidrográfica cedente, y 2) el uso del agua del trasvase se enmarca en la tendencia global neoliberal de apropiación de los recursos naturales donde los intereses privados se imponen sobre el social general en una relación de poder asimétrica.

En definitiva, el insuficiente cumplimiento de la DMA dentro del marco legislativo español iniciado por su tardía transposición y continuado por la falta de compromiso con el mandamiento de la preferencia medioambiental en la gestión del agua que la directiva impone influye de manera directa en el trasvase Tajo-Segura. En referencia a la sostenibilidad ambiental, se ha seguido privilegiando a la demanda económica y esto ha causado que la detracción de agua haya impedido disponer de unos verdaderos caudales ecológicos para el Tajo. Tampoco se recuperan los costes de la infraestructura tal y como marca la DMA, dado que en las tarifas del agua no se incluyen. De esta forma, se favorecen los intereses privados de la cuenca receptora al acceder al agua trasvasada con un precio menor al que debería fijarse y, por lo tanto, estar así indirectamente subvencionada. Finalmente, se ha permitido un mercado de agua entre particulares que funciona de manera opaca 
y que permite en periodos de sequía, donde el trasvase normal está muy limitado al tener que cumplir con lo dictado por la DMA, la cesión de agua entre cuencas.

La prioridad debería ser satisfacer la demanda de agua dulce como necesidad de abastecimiento para toda la población y evitar los impactos al medio ambiente. Sin embargo, es evidente que las autoridades están principalmente preocupadas por satisfacer las demandas de agua que tienen que ver con la producción económica aun a sabiendas de no cumplir plenamente con la legislación europea. Se necesitan soluciones imaginativas para buscar alternativas y así respetar el espíritu de la DMA de prevalencia de la protección del agua y anteponer el interés público social al privado, algo que no siempre está al alcance del ámbito político actual. Por esta razón, futuros trabajos académicos deberán seguir abordando esta cuestión.

\section{Referencias}

Baeza Sanz, D. (20I3). El estado ecológico de la cuenca del Tajo. En Larraz, B. y Cano, A. (Coords.). El río Tajo, lecciones del pasado para un futuro mejor (pp. 79-90). Toledo: Ledoria.

Bakker, K. (2002). From state to market? Water mercantilización in Spain. Environment and Planning A, 34 (I), $767-790$. DOI: 10.1068/a3425.

Bauer, C. J. (20I0). El abanico de bancos de aguas en las Américas. Estudios de Economía Aplicada, 28 (2), 227-236.

Barreira, A. (2008). La gestión de las cuencas hispano-portuguesas: El Convenio de Albufeira. Fundación Nueva Cultura del Agua. Recuperado de: http://www.iidma.org/attachments/Publicaciones/hispanoportugal.pdf (7.4.2020).

Berbel, J., Gutiérrez Martín, C. y Giannocaro, G. (2016). Una visión realista de los mercados de derechos de agua. En Gómez, J.A. y Calatrava, J. (Coords). Los mercados de agua en España (pp. 4I I-426). Almería: Cajamar.

Bolinches, A., De Stefano, L. y Paredes-Arquiola, J. (2020). Designing river water quality policy interventions with scarce data: the case of the Middle Tagus Basin, Spain. Hydrological Sciences Journal, 65 (5), 749-762. DOI: I0.1080/02626667.2019.17089I5.

Box Amorós, M. (1988). El trasvase Tajo-Segura. En Gil,A. y Morales, A. (Eds.) Demanda y economía del agua en España (pp. 277-286). Alicante: Universidad de Alicante.

Box Amorós, M. y Morales Gil, A. (1994). Consecuencias socioeconómicas y medioambientales de los trasvases de agua en España (1978-1992). Investigaciones Geográficas, 22, 25-36.

Bromley, D.W. ( 1 992). Problemas de la gestión del agua y del suelo: una perspectiva institucional. En Aguilera, F. (Ed.). Economía del agua (Pp. III-I37). Madrid: MAPA.

Calatrava, J. y Martínez-Granados, D. (20I2). El valor de uso del agua en el regadío de la cuenca del Segura y en las zonas regables del trasvase Tajo-Segura. Economía Agraria y Recursos Naturales, I 2 (I), p. 5-32.

Calvo García-Tornel, F. (2002). Plan Hidrológico Nacional y déficit estructural en la cuenca del Segura. En Gil, A. y Morales,A. (Eds.) Insuficiencias Hídricas y Plan Hidrológico Nacional (pp. 3 I 9-346).Alicante: Caja de Ahorros del Mediterráneo.

Castree, N. (2008). Neoliberalising nature: processes, effects, and evaluations. Environment and Planning A, 40 (I), I 53 173. DOI: 10.1068/a39100.

Comisión Europea (2019). Informe sobre la aplicación de los Planes Hidrológicos de Cuenca de la Directiva Marco del Agua, Estado miembro: España. Recuperado de: https://ec.europa.eu/environment/water/water-framework/ pdf/4th_report/MS\%20annex\%20-\%20Spain_es.pdf (I3.II.2019)

Confederación Hidrográfica del Segura (CHS) (2020). Históricos. Recuperado de: https://www.chsegura.es/chs/ cuenca/infraestructuras/postrasvaseTajoSegura/historicos.html (23.I.2020).

Del Moral, L., Pita, M. F., Pedregal, B., Hernández-Mora, N.y Limones, N. (20I4). Current paradigms in the management of water: Resulting information needs. Publicationes Instituti Geographici Universitatis Tartuensis, I I 0, 2 I-3 I.

Delgado Piqueras, F. (2002). La gestión de las cuencas hidrográficas hispano-portuguesas: el convenio de Albufeira de 1998. Revista de Administración Pública, 158, 301-324.

Diario ABC (25-6-207). Castilla-La Mancha manda un informe a la UE sobre el impacto negativo del trasvase. Recuperado de: https://www.abc.es/espana/castilla-la-mancha/abci-castilla-la-mancha-manda-informe-sobreimpacto-negativo-trasvase-2017062521I7_noticia.html (22.1.2020).

Dufour, S. y Piegay, H. (2009). From the myth of a lost paradise to targeted river restoration: forget natural references and focus on human benefits. River Research and Application, 25 (5), 568-8I. DOI: I0.1002/rra.I 239.

Escribano Frances, G., Quevauviller, P., San Martín González, E. y Vargas Amelín, E. (2017) Climate change policy and water resources in the EU and Spain. A closer look into the Water Framework Directive. Environmental Science \& Policy, 69, I-I2. DOI: 10.1016/j.envsci.2016.12.006.

Espinosa, S. (2008). Regulación internacional del agua dulce. Cizur Menor:Aranzadi. 
Ferreira, M.T. (2013). Calidad ecológica en la Demarcación Hidrográfica del Tajo: una perspectiva integrada. En Baeza, D. et al. El Tajo. Historia de un río ignorado (pp. I45-I7I). Zaragoza: Fundación Nueva Cultura del Agua.

Gallego Bernad, M. S. (20I3a). El abastecimiento de Madrid y el trasvase Tajo-Segura en la planificación y gestión de la Demarcación Hidrográfica del Tajo. En Baeza, D. et al. El Tajo. Historia de un río ignorado (pp. 35-88). Zaragoza: Fundación Nueva Cultura del Agua.

Gallego Bernad, M. S. (20I3b). El coste del agua trasvasada del Tajo frente a la desalación En Larraz, B. y Cano, A. (Coords.). El río Tajo, lecciones del pasado para un futuro mejor (pp. 57-70). Toledo: Ledoria.

Garrido Colmenero, A. (1999). La economía del agua en España. Entre lo público y lo privado, la difícil búsqueda del interés general. Arbor, CLXIV, 2I7-239.

Gil Meseguer, E., Martínez Medina, R. y Gómez Espín, J. M. (2018). El trasvase Tajo-Segura (I979-2017). Actuaciones para su futuro en España. Tecnología y Ciencias del Agua, 9 (2), 192-209. DOI: 10.24850/j-tyca-20 I8-02-08.

Gil Olcina, A. (1992). Desequilibrios hidrográficos en España y trasvases a la vertiente mediterránea: utopías y realidades. Investigaciones Geográficas, 10, 7-22.

Gil Olcina, A. (1994). Conflictos económicos sobre trasvases de agua en España. Investigaciones Geográficas, I3, I7-28.

Gómez Espín, J. M. (Coord.) (20I7). El trasvase Tajo-Segura. Propuestas para su continuidad y futuro. Saarbrücken: Editorial Académica Española.

Gómez Espín, J. M., López, J. A. y Montaner, M. E. (Coords.) (20I I). Modernización de regadíos: sostenibilidad social y económica. La singularidad de los regadíos del Trasvase Tajo-Segura. Murcia: Universidad de Murcia.

Gómez Mendoza, J. y Mata Olmo, R. (1999). Abastecimiento urbano, regadíos, trasvases y planificación en la cuenca del Tajo. En Gil,A.y Morales,A. (Eds.). Los usos del agua en España (pp. 30l-336).Alicante: Caja de Ahorros del Mediterráneo y Universidad de Alicante.

Gómez Nicola, G. (20I3). Ictiofauna del Tajo. Los peces como indicadores biológicos. En Larraz, B.y Cano,A. (Coords.). El río Tajo, lecciones del pasado para un futuro mejor (pp. I57-I72). Toledo: Ledoria.

González Rojas, D. (2018). La gestión de las cuencas hidrográficas en España: avances y carencias del segundo ciclo de planificación. Agua y Territorio, I I, I23-136. DOI: 10.1756 I/at. I I.3027.

Harvey, D. (2003). The new imperialism. Oxford, UK: Oxford University Press.

Hering, D., Borja, A., Cartensen, J., Carvalho, L., Elliott, M., Feld, C. K., Heiskanen, A. S., Johnson, R. K., Moe, J., Pont, D., Solheim, A. L. y van de Bund,W. (2010). The European Water Framework Directive at the age of 10:A critical review of the achievements with recommendations for the future. Science of the Total Environment, 408, 400740I9. DOI: 10.1016/j.scitotenv.2010.05.031.

Hernández-Mora, N. (2012). La planificación hidrológica y la Directiva Marco del Agua en España en el periodo 20002012. Fundación Nueva Cultura del Agua. Recuperado de: https://www.fnca.eu/guia-nueva-cultura-del-agua/ images/documents/Planificaci\%C3\%B3n/La-planificacion-hidrologica-Tajo.pdf (8.4.2020).

Hernández-Mora, N. (20I3). La planificación hidrológica y la Directiva Marco del Agua en España en el periodo 200020 12: ¿Hemos avanzado? En Larraz, B. y Cano,A. (Coords.) El río Tajo, lecciones del pasado para un futuro mejor (pp. 39-56). Toledo: Ledoria.

Hernández-Mora, N. (2019).A vueltas con el trasvase Tajo-Segura. Fundación Nueva Cultura del Agua. Recuperado de: https://fnca.eu/73-boletin/titulos/567-a-vueltas-con-el-trasvase-tajo-segura (I6.12.2019).

Hernández-Mora, N. y del Moral, L. (2016). Disfuncionalidades de los mercados de agua en España. En Gómez, J.A.y Calatrava, J. (Coords). Los mercados de agua en España (pp. 427-460).Almería: Cajamar.

Hernández-Mora, N., Del Moral Ituarte, L., La Roca, F., La Calle, A. y Schmidt, G. (20I4). Interbasin water transfers in Spain: Interregional conflicts and governance responses. En Schneier-Madanes, G. (Ed.). Globalized Water: A Question of Governance (pp. I75-194). Dordrecht: Springer.

Hill, G., del Moral, L, Paneque, P., Pedregal, B., Spash, C. y Urama, K. (2002). Evaluation practices in water project decision-making processes comparative analysis of Alqueva (Portugal) and Ebro River Transfer (Spain). En del Moral, L. (Coord.). La Directiva Marco del Agua: realidades y futuros (pp. 62-69). Sevilla: Universidad Pablo de Olavide, Universidad de Sevilla y Fundación Nueva Cultura del Agua.

Juárez Sánchez-Rubio, C. (1991): Planificación hidrológica y desarrollo económico: el Trasvase Tajo-Segura.Alicante: Instituto de Cultura Juan Gil Albert.

Kahnna, P. (20I7). Conectografia (P. Hermida, trad.). Barcelona: Espasa. (Obra original publicada en 20I6).

Kaika, M. (2003). The WFD: A new directive for a changing social, political and economic European framework. European Planning Studies, II (3), 299-3 I6. DOI: I0. I080/096543 I0303640.

Kallis, G. y Butler, D. (200I). The EU water framework directive: measures and implications. Water Policy, 3, I25-I 42.

Kallis, G., Videira, N., Antunes, P. Guimarães Pereira, A., Spash, C.L., Coccossis, H., Corral Quintana, S., del Moral, L., Hatzilacou, D., Lobo, G., Mexa, A., Paneque, P., Pedregal Mateos, B. y Santos, R. (2006). Participatory methods for water resources planning. Environment and Planning C: Government and Policy, 24, 2I 5-234. DOI: 10.1068/ c04102s.

La Calle Marcos, A. (2015). Nuevos enfoques institucionales para la gestión del agua: Directiva Marco de Agua. En Del Moral, L.,Arrojo, P. y Herrera, T. (Coords.). El agua: Perspectiva ecosistémica y gestión integrada (pp. I6-48). Zaragoza: Fundación Nueva Cultura del Agua.

Martínez Fernández, J. y Esteve Selma, M. A. (2000). Sequía estructural y algunas externalidades ambientales en los regadíos de la cuenca del Segura. Ingeniería del Agua, 7 (2), I65-172.

Martínez Paz, J. M., Perni, A., Ruiz Campuzano, F. y Pellicer Martínez, F. (2016).Valoración económica de los fallos de 
suministro en los regadíos de la cuenca del Segura. Revista Española de Estudios Agrosociales y Pesqueros, 244, 35-67

McCaffrey, S. C. (2018). Introduction: The Path to the UN Watercourses Convention and Beyond. En Boisson de Cahzourn, L., Moïse, M.,Tignino, M. y Sangbana, K. (Eds.) The UN Convention on the Law of the Non-Navigational Uses of International Watercourses. A Commentary (pp. I- I8). Oxford: Oxford University Press.

Melgarejo Moreno, J. (1997). El trasvase Tajo-Segura en el centro de la tormenta política de la transición. Áreas, I7, I29-I44.

Melgarejo Moreno, J. (2000). El balance económico del trasvase Tajo-Segura. Investigaciones Geográficas, 24, 69-95.

Ministerio para la Transición Ecológica (MITECO) (2019). Textos legislativos. Recuperado de: https://www.miteco.gob. es/es/agua/temas/planificacion-hidrologica/marco-del-agua/LegislacionDMA.aspx (7.12.2019).

Morales Gil, A., Rico Amorós, A. M. y Hernández Hernández, M. (2005). El trasvase Tajo-Segura. Observatorio Medioambiental, 8, 73-II0.

Morote, A. F., Olcina, J.y Rico, A. M. (2017). Challenges and proposals for socio-ecological sustainability of the TagusSegura aqueduct (Spain) under climate change. Sustainability, 9 (I I), I-24. DOI: 10.3390/su9 I I 2058.

Morote Seguido, A. F., Olcina Cantos, J. y Rico Amorós, A. M. (20I8). Un trasvase cuestionado: El Tajo-Segura. Repercusiones socio-económicas en el sureste español e incertidumbre ante el cambio climático. Revista de Estudios Regionales, II3, 29-70.

Morote Seguido, A. F. y Rico Amorós, A. M. (2018). Perspectivas de funcionamiento del trasvase Tajo-Segura (España): efectos de las nuevas reglas de explotación e impulso de la desalinización como recurso sustitutivo. Boletín de la Asociación de Geógrafos Españoles, 79, 2754.

Moss, B. (2008). The Water Framework Directive: Total environment or political compromise? Science of the Total Environment, 400 (I-3), 32-4I. DOI: I0.1016/j.scitotenv.2008.04.029.

Movilla Pateiro, L. (20I4). La entrada en vigor de la Convención sobre el Derecho de los Usos de los Cursos de Agua Internacionales para Fines Distintos de la Navegación. Revista Española de Derecho Internacional, 66, 3I 2-3 I6.

Naredo,J.M. (2007). Lo público y lo privado, la planificación y el mercado, en la encrucijada actual de la gestión del agua en España. Fundación Nueva Cultura de la Política deAguas. Recuperado de: https://www.paralelo36andalucia. com/wp-content/20 I I/09/agua2.pdf (13.4.2020).

Oficina de Publicaciones de la Unión Europea (2012). Sentencia del Tribunal de Justicia (Sala Sexta) de 4 de octubre de 2012. Comisión Europea contra Reino de España. Incumplimiento de Estado Directiva 2000/60/ CE. Recuperado de: https://op.europa.eu/es/publication-detail/-/publication/40db7d43-0f47-4f7b-94289507/96e464d/language-es (8.4.2020).

Oñate, J. J. (2016). Mercado de agua y medio ambiente En Gómez, J. A. y Calatrava, J. (Coords) Los mercados de agua en España (pp. 179-207).Almería: Cajamar.

Organización de Naciones Unidas (ONU) (2020). Agua. Disponible en: https://www.un.org/es/sections/issues-depth/ water/index.html (7.4.2020).

Palomo Hierro, S. y Gómez Limón, J.A. (20l4). El papel de los mercados como instrumento para la reasignación del agua en España. Agua y Territorio, I (2), 78-92.

Palomo Hierro, S. y Gómez Limón, J. A. (20I6). Actividad de los mercados formales de agua en España (I999-20|4). En Gómez, J.A. y Calatrava,J. (Coords.). Los mercados del agua en España. Presente y perspectivas (pp. I27-I58). Almería: Cajamar.

Pérez Crespo,A. (2009). Los orígenes y puesta en marcha del trasvase Tajo-Segura: una crónica personal. Murcia: Fundación Instituto Euromediterráneo del Agua.

Pita, M. F., Del Moral, L., Pedregal, B., Limones, N. y Hernández-Mora, N. (20I4). Nuevos paradigmas en la gestión de recursos y riesgos hídricos: datos de información necesarios para una gestión integrada del agua. Boletín de la Asociación de Geógrafos Españoles, 65, 5I7-540.

Rico Amorós, A.M., Saurí, D., Olcina-Cantos, J. y Vera Rebollo, F. (2013). Beyond megaprojects? Water alternatives for mass tourism in coastal Mediterranean Spain. Water Resources Management, 27 (2), 553-565. DOI: I0.1007/ sII269-0|2-020|-3.

Salinas Palacio, D. (20I3). El convenio de la Albufeira y el plan de cuenca del Tajo. ¿Qué implicaciones para el trasvase Tajo-Segura? Revista de Administración Pública, I58, 30 I-324.

Salinas Palacios, D. y Loyer, B (2013). Eau et pouvoir locaux en Espagne (Géopolitique de l'eau : Les enjeux de l'or bleu). Diplomatie - Les grands dossiers, 15, 36-39.

Sandoval Rodríguez, J. M. (1989). El trasvase Tajo-Segura: solución al desequilibrio hidrológico. Madrid: Nuevos Enfoques.

San Martín González, E. (20I I). Un análisis económico de los trasvases de agua intercuencas: el trasvase Tajo-Segura (Tesis doctoral). UNED. Recuperado de: http://e-spacio.uned.es/fez/view.php?pid=tesisuned:CiencEcoEmpEsanmartin (I3.4.2020)

San Martín González, E., Larraz Iribas, B., Hernández-Mora, N. y Gallego Bernad, M. S. (20I5). La gestión insostenible del río Tajo. Biblio 3W. Revista Bibliográfica de Geografia y Ciencias Sociales, XX, I.I33.

Saurí Pujol, D. y del Moral Ituarte, L. (200I). Recent development in Spanish water policy.Alternatives and conflicts at the end of the hydraulic age. Geoforum, 32 (3), 35I-362. DOI: I0.10I6/S00I6-7I85(00)00048-8.

Swyngedouw, E. (2007). Dispossessing $\mathrm{H}_{2} \mathrm{O}$ :The contested terrain of water privatization. En Heynen, N., McCarthy, J., Prudham, S. y Robbins, P. (Eds.). Neoliberal Environments: False promises and unnatural consequences (pp. 5I-62). Nueva York: Routledge. 
Tortajada, C. (2016). Policy dimensions of development and financing of water infrastructure:The cases of China and India. Enviromental Science \& Policy, 64, I77-187. DOI: I0.1016/j.envsci.2016.07.00 I.

World Wildlife Fund (WWF) (17-12-2019). Celebramos que la Comisión Europea respalde la Directiva Marco del Agua. Recuperado de: https://www.wwf.es/informate/actualidad/?52980/Celebramos-que-la-ComisionEuropea-respalde-la-Directiva-Marco-del-Agua (9.4.2020).

Yela, J. L., (2013). Invertebrados del río Tajo a su paso por Toledo: entre la realidad y la especulación en el contexto de la crisis ambiental actual. En Larraz, B. y Cano,A. (Coords.). El río Tajo, lecciones del pasado para un futuro mejor (pp. I23-156). Toledo: Ledoria.

Zhuang,W.(2016). Eco-environmental impact of inter-basin water transfer projects: a review. Environmental Science and Pollution Research, 23 (I3), I 2867-I2879. DOI: I0.1007/s I |356-0 I6-6854-3. 


\section{RELACIONES INTERNACIONALES}

Revista académica cuatrimestral de publicación electrónica

Grupo de Estudios de Relaciones Internacionales (GERI)

Universidad Autónoma de Madrid, España

https://revistas.uam.es/relacionesinternacionales

ISSN 1699 - 3950

f facebook.com/RelacionesInternacionales

3. twitter.com/RRInternacional 